\title{
Positive solutions for a second-order $p$-Laplacian impulsive boundary value problem
}

\author{
Youzheng Ding ${ }^{1,3^{*}}$ and Donal O'Regan ${ }^{2}$
}

\section{"Correspondence:}

dingyouzheng@139.com

'Department of Mathematics,

Shandong Jianzhu University, Jinan,

Shandong 250101, China

${ }^{3}$ School of Mathematics, Shandong

University, Jinan, Shandong 250100

China

Full list of author information is available at the end of the article

\begin{abstract}
In this work, we study the existence and multiplicity of positive solutions for a second-order $p$-Laplacian boundary value problem involving impulsive effects. We establish our main results via Jensen's inequality, the first eigenvalue of a relevant linear operator and the Krasnoselskii-Zabreiko fixed point theorem. Some examples are presented to illustrate the main results.
\end{abstract}

MSC: 34B15; 34B18; 34B37;45G15;45M20

Keywords: impulsive boundary value problem; -Laplacian; fixed point theorem; positive solution; eigenvalue; Jensen inequality

\section{Introduction}

Second-order differential equations with the $p$-Laplacian operator arise in modeling some physical and natural phenomena and can occur, for example, in non-Newtonian mechanics, nonlinear elasticity, glaciology, population biology, combustion theory, and nonlinear flow laws, see [1,2]. Recently, many cases of the existence and multiplicity of positive solutions for boundary value problems of differential equations with the $p$-Laplacian operator have appeared in the literature. For details, see, for example, [3-13] and the references therein.

In [3], Lian and Ge investigated the Sturm-Liouville-like boundary value problem

$$
\left\{\begin{array}{l}
\left(\varphi_{p}\left(u^{\prime}(t)\right)^{\prime}+f(t, u(t))=0, \quad 0<t<1,\right. \\
u(0)-a u^{\prime}(\xi)=0, \quad u(1)+\beta u^{\prime}(\eta)=0,
\end{array}\right.
$$

and by virtue of Krasonsel'skii's fixed point theorem, they obtained the existence of positive solutions and multiple positive solutions under suitable conditions imposed on the nonlinear term $f \in C([0,1] \times[0,+\infty),[0,+\infty))$.

In [6], Xu et al. studied the existence of multiple positive solutions for the following boundary value problem with the $p$-Laplacian operator and impulsive effects

$$
\left\{\begin{array}{l}
\left(\phi_{p}\left(u^{\prime}(t)\right)^{\prime}+q(t) f(t, u(t))=0, \quad t \neq t_{k}, 0<t<1,\right. \\
\left.\Delta u\right|_{t=t_{k}}=I_{k}\left(u\left(t_{k}\right)\right), \quad k=1,2, \ldots, m, \\
a u(0)-b u^{\prime}(0)=\sum_{i=1}^{l} \alpha_{i} u\left(\xi_{i}\right), \quad u^{\prime}(1)=\sum_{i=1}^{l} \beta_{i} u^{\prime}\left(\xi_{i}\right),
\end{array}\right.
$$


where the nonlinear term may be singular on $u=0$. The main tools are fixed point index theorems for compact maps in Banach spaces. They stated the proofs by considering an approximating completely continuous operator.

In [7], Feng studied an integral boundary value problem of fourth-order $p$-Laplacian differential equations involving the impulsive effect $\left.\Delta y^{\prime}\right|_{t=t_{k}}=-I_{k}\left(y\left(t_{k}\right)\right), k=1,2, \ldots, m$. Using a suitably constructed cone and fixed point theory for cones, the existence of multiple positive solutions was established. Furthermore, upper and lower bounds for these positive solutions were given.

Motivated by the above works, in this paper, we investigate the existence and multiplicity of positive solutions for the second-order $p$-Laplacian boundary value problems involving impulsive effects

$$
\left\{\begin{array}{l}
\left(\varphi_{p}\left(u^{\prime}(t)\right)\right)^{\prime}=-f(t, u(t)), \quad 0<t<1, t \neq t_{k}, k=1,2, \ldots, m, \\
\left.\Delta u\right|_{t=t_{k}}=I_{k}\left(u\left(t_{k}\right)\right), \quad k=1,2, \ldots, m, \\
\left.\Delta u^{\prime}\right|_{t=t_{k}}=0, \quad k=1,2, \ldots, m, \\
u(0)=u^{\prime}(1)=0,
\end{array}\right.
$$

where $J=[0,1], J^{\prime}=J \backslash\left\{t_{1}, t_{2}, \ldots, t_{m}\right\}, t_{k}(k=1,2, \ldots, m$, where $m$ is a fixed positive integer $)$ are fixed points with $0<t_{1}<t_{2}<\cdots<t_{k}<\cdots<t_{m}<1$; $\varphi_{p}(t)$ is the $p$-Laplacian operator, i.e., $\varphi_{p}(t)=|t|^{p-2} t, p>1,\left(\varphi_{p}\right)^{-1}=\varphi_{q}, p^{-1}+q^{-1}=1 ;\left.\Delta u\right|_{t=t_{k}}$ denotes the jump of $u(t)$ at $t=t_{k}$, i.e., $\left.\Delta u\right|_{t=t_{k}}=u\left(t_{k}^{+}\right)-u\left(t_{k}^{-}\right)$, where $u\left(t_{k}^{+}\right)$and $u\left(t_{k}^{-}\right)$represent the right-hand limit and left-hand limit of $u(t)$ at $t=t_{k}$, respectively. In addition, we suppose that $I_{k} \in C([0,+\infty),[0,+\infty))$, $f \in C([0,1] \times[0,+\infty),[0,+\infty))$.

The main features of this paper are as follows. Firstly, we convert the boundary value problem (1.3) into an equivalent integral equation so that we can construct a special cone. Next, we consider impulsive effect as a perturbation to the corresponding problem without the impulsive terms, so that we can construct an integral operator for an appropriate linear Robin boundary value problem and obtain its first eigenvalue and eigenfunction, which are used in the proofs of main theorems by Jensen's inequalities. Finally, employing the Krasnoselskii-Zabreiko fixed point theorem, we establish the existence and multiplicity of positive solutions of (1.3). Although our problem (1.3) merely involves Robin boundary conditions, our methods are different from those in $[3,6,7]$, and our main results are optimal.

This paper is organized as follows. Section 2 contains some preliminary results. Section 3 is devoted to the existence and multiplicity of positive solutions for (1.3). Section 4 contains some illustrative examples.

\section{Preliminaries}

Let $P C[J, R]:=\left\{u \mid u: J \rightarrow R\right.$ is continuous at $t \neq t_{k}, u\left(t_{k}^{-}\right)=u\left(t_{k}\right)$ and $u\left(t_{k}^{+}\right)$exist, $k=1,2, \ldots$, $m\}$. Then $P C[J, R]$ is a Banach space with norm $\|u\|=\max _{t \in[0,1]}|u(t)|$. We denote $B_{r}:=$ $\{u \in P C[J, R]:\|u\|<r\}$ for $r>0$ in the sequel.

A function $u \in P C[J, R] \cap C^{2}\left(J^{\prime}, R\right)$ is called a solution of (1.3) if it satisfies the boundary value problem (1.3). 
Lemma 2.1 (see [4]) Let $f$ and $I_{k}$ be as in (1.3). Then the problem (1.3) is equivalent to

$$
u(t)=\int_{0}^{t} \varphi_{q}\left(\int_{s}^{1} f(\tau, u(\tau)) \mathrm{d} \tau\right) \mathrm{d} s+\sum_{t_{k}<t} I_{k}\left(u\left(t_{k}\right)\right) .
$$

It is clear that $u^{\prime}(t)=\varphi_{q}\left(\int_{t}^{1} f(\tau, u(\tau)) \mathrm{d} \tau\right)>0, t \in J^{\prime}$ and $I_{k}>0$, which implies that $u(t)$ is increasing on $[0,1]$. Furthermore, for given $s_{1}, s_{2} \in J^{\prime}$ with $s_{1} \leq s_{2}$, we have $u^{\prime}\left(s_{2}\right) \leq u^{\prime}\left(s_{1}\right)$. Hence, $u^{\prime}(t)$ is nonincreasing on $J^{\prime}$, and thus

$$
\frac{u(1)-u(0)}{1} \leq \frac{u(t)-u(0)}{t}, \quad t \in(0,1]
$$

i.e., $u(t) \geq t u(1)=t\|u\|$. Therefore,

$$
u(t) \geq t\|u\|, \quad \forall t \in[0,1], \quad \text { in particular, } \quad u(t) \geq t_{1}\|u\|, \quad \forall t \in\left[t_{1}, t_{m}\right] .
$$

We denote $P$ by

$$
P:=\{u \in P C[J, R]: u(t) \geq t\|u\|, t \in[0,1]\} .
$$

Then $P$ is a cone on $P C[, R]$.

Define an operator $A: P \rightarrow P C[, R]$

$$
\begin{aligned}
A u(t) & :=\int_{0}^{t} \varphi_{q}\left(\int_{s}^{1} f(\tau, u(\tau)) \mathrm{d} \tau\right) \mathrm{d} s+\sum_{t_{k}<t} I_{k}\left(u\left(t_{k}\right)\right) \\
& =\int_{0}^{t} \varphi_{q}\left(\int_{s}^{1} f(\tau, u(\tau)) \mathrm{d} \tau\right) \mathrm{d} s+\sum_{k=1}^{m} H\left(t, t_{k}\right) I_{k}\left(u\left(t_{k}\right)\right),
\end{aligned}
$$

where

$$
H\left(t, t_{k}\right)= \begin{cases}1, & t_{k}<t \\ 0, & t_{k} \geq t\end{cases}
$$

Clearly, the operator $A$ is a completely continuous operator, and the existence of positive solutions for (1.3) is equivalent to that of positive fixed points of $A$. Moreover, it is easy to see $A(P) \subset P$ by $(2.2)$.

In what follows, we consider the following eigenvalue problem:

$$
\left\{\begin{array}{l}
-u^{\prime \prime}=\lambda u, \quad t \in[0,1] \\
u(0)=u^{\prime}(1)=0
\end{array}\right.
$$

where $\lambda$ is a parameter. We easily know that (2.4) has a nontrivial solution if $\lambda>0$. Furthermore, we have

$$
u(t)=c_{1} \cos \sqrt{\lambda} t+c_{2} \sin \sqrt{\lambda} t
$$


where $c_{1}, c_{2}$ are constants and $c_{1}^{2}+c_{2}^{2} \neq 0 . u(0)=u^{\prime}(1)=0$ implies that $\cos \sqrt{\lambda}=0$, and thus $\lambda=\left(-\frac{\pi}{2}+k \pi\right)^{2}, k=1,2 \ldots \lambda_{1}^{-1}:=\left(-\frac{\pi}{2}+1 \times \pi\right)^{2}=\frac{\pi^{2}}{4}$ and $\sin \left(\frac{\pi t}{2}\right)$ are called the first eigenvalue and the corresponding eigenfunction associated with $\lambda_{1}$, respectively. Consequently, it is easy to have the following result:

Lemma 2.2 If $\psi(t):=\sin \left(\frac{\pi t}{2}\right)$, then

$$
\int_{0}^{1} G(t, s) \psi(t) \mathrm{d} t=\frac{4}{\pi^{2}} \psi(s)
$$

where $G(t, s)=\min \{t, s\}, t, s \in[0,1]$.

Lemma 2.3 (see [14]) Let E be a real Banach space and $W$ a cone of E. Suppose $A:\left(\bar{B}_{R} \backslash\right.$ $\left.B_{r}\right) \cap W \rightarrow W$ is a completely continuous operator with $0<r<R$. If either

(1) $A u \not \leq u$ for each $\partial B_{r} \cap W$ and $A u \nsupseteq u$ for each $\partial B_{R} \cap W$ or

(2) $A u \nsupseteq u$ for each $\partial B_{r} \cap W$ and $A u \not \leq u$ for each $\partial B_{R} \cap W$, then $A$ has at least one fixed point in $\left(B_{R} \backslash \bar{B}_{r}\right) \cap W$.

Lemma 2.4 (Jensen's inequalities, see [10]) Let $\theta>0, n \geq 1, a_{i} \geq 0(i=1,2, \ldots, n)$, and $\varphi \in C([a, b],[0,+\infty))$. Then

$$
\begin{aligned}
& \left(\int_{a}^{b} \varphi(t) \mathrm{d} t\right)^{\theta} \leq(b-a)^{\theta-1} \int_{a}^{b}(\varphi(t))^{\theta} \mathrm{d} t \quad \text { and } \\
& \left(\sum_{i=1}^{n} a_{i}\right)^{\theta} \leq n^{\theta-1} \sum_{i=1}^{n} a_{i}^{\theta}, \quad \forall \theta \geq 1, \\
& \left(\int_{a}^{b} \varphi(t) \mathrm{d} t\right)^{\theta} \geq(b-a)^{\theta-1} \int_{a}^{b}(\varphi(t))^{\theta} \mathrm{d} t \quad \text { and } \\
& \left(\sum_{i=1}^{n} a_{i}\right)^{\theta} \geq n^{\theta-1} \sum_{i=1}^{n} a_{i}^{\theta}, \quad \forall 0<\theta \leq 1 .
\end{aligned}
$$

\section{Main results}

Let $p^{*}:=\max \{1, p-1\}, p^{*}:=\min \{1, p-1\}, \kappa_{1}:=2^{p^{s}-1}, \kappa_{2}:=(2 m)^{p^{n}-1}, \kappa_{3}:=2^{p^{*}-1}, \kappa_{4}:=$ $(2 m)^{p^{*}-1}, \kappa_{5}:=2^{\frac{p p^{p}}{p-1}-2}, \kappa_{6}:=(4 m)^{p^{*}-1}$. We now list our hypotheses.

(H1) There exist $r>0$ and $a_{1} \geq 0, a_{2} \geq 0$ satisfying

$$
a_{1}^{\frac{p^{*}}{p-1}} \kappa_{1}+\frac{\pi^{2}}{4} a_{2}^{p^{*}} t_{1}^{p^{*}} \kappa_{2} \sum_{k=1}^{m} \cos \left(\frac{\pi t_{k}}{2}\right)>\frac{\pi^{2}}{4}
$$

such that

$$
f(t, y) \geq a_{1} y^{p-1}, \quad I_{k}(y) \geq a_{2} y, \quad \forall t \in[0,1], 0<y<r .
$$

(H2) There exist $c>0$ and $b_{1} \geq 0, b_{2} \geq 0$ satisfying

$$
b_{1}^{2}+b_{2}^{2} \neq 0, \quad b_{1}^{\frac{p^{n}}{p-1}} \kappa_{5}+\frac{\pi b_{2}^{p^{*}} \kappa_{6} \sum_{k=1}^{m} \cos \left(\frac{\pi t_{k}}{2}\right)}{2 \int_{0}^{1} t^{p^{n}} \sin \left(\frac{\pi t}{2}\right) \mathrm{d} t}<\frac{\pi^{2}}{4}
$$


such that

$$
f(t, y) \leq b_{1} y^{p-1}+c, \quad I_{k}(y) \leq b_{2} y+c, \quad \forall t \in[0,1], y \geq 0 .
$$

(H3) There exist $c>0$ and $a_{3} \geq 0, a_{4} \geq 0$ satisfying

$$
a_{3}^{\frac{p^{*}}{p-1}} \kappa_{1}+\frac{\pi^{2}}{4} a_{4}^{p^{*}} t_{1}^{p^{*}} \kappa_{2} \sum_{k=1}^{m} \cos \left(\frac{\pi t_{k}}{2}\right)>\frac{\pi^{2}}{4},
$$

such that

$$
f(t, y) \geq a_{3} y^{p-1}-c, \quad I_{k}(y) \geq a_{4} y-c, \quad \forall t \in[0,1], y \geq 0 .
$$

(H4) There exist $r>0$ and $b_{3} \geq 0, b_{4} \geq 0$ satisfying

$$
b_{3}^{2}+b_{4}^{2} \neq 0, \quad b_{3}^{\frac{p^{n}}{p-1}} \kappa_{5}+\frac{\pi b_{4}^{p^{n}} \kappa_{6} \sum_{k=1}^{m} \cos \left(\frac{\pi t_{k}}{2}\right)}{2 \int_{0}^{1} t^{p^{n}} \sin \left(\frac{\pi t}{2}\right) \mathrm{d} t}<\frac{\pi^{2}}{4},
$$

such that

$$
f(t, y) \leq b_{3} y^{p-1}, \quad I_{k}(y) \leq b_{4} y, \quad \forall t \in[0,1], 0<y<r .
$$

(H5) There exists $\rho>0$ such that $0 \leq y \leq \rho$, and $t \in[0,1]$ implies

$$
f(t, y) \leq \eta^{p-1} \rho^{p-1}, \quad I_{k}(y) \leq \eta_{k} \rho,
$$

where $\eta, \eta_{k} \geq 0$ and $0<\eta+\sum_{k=1}^{m} \eta_{k} \leq 1$.

(H6) There exists $\rho>0$ such that $t_{1} \rho \leq y \leq \rho$, and $t \in[0,1]$ implies

$$
f(t, y) \geq \eta^{p-1} \rho^{p-1}, \quad I_{k}(y) \geq \eta_{k} \rho,
$$

where $\eta, \eta_{k} \geq 0,\left(\eta p^{-1}(p-1)\left(1-t_{1}\right)^{p /(p-1)}+\sum_{k=1}^{m} \eta_{k}\right)>1$.

Theorem 3.1 Suppose that (H1)-(H2) are satisfied. Then (1.3) has at least one positive solution.

Proof If (H1) is satisfied, then we obtain $u \nsupseteq A u$ for all $u \in P \cap \partial B_{r}$. Indeed, if the claim is false, there is a $u \in P \cap \partial B_{r}$ such that $u \geq A u$, i.e.,

$$
u(t) \geq \int_{0}^{t} \varphi_{q}\left(\int_{s}^{1} f(\tau, u(\tau)) \mathrm{d} \tau\right) \mathrm{d} s+\sum_{k=1}^{m} H\left(t, t_{k}\right) I_{k}\left(u\left(t_{k}\right)\right) .
$$

Now apply Lemma 2.4 to obtain

$$
\begin{aligned}
u^{p^{*}}(t) & \geq\left[\int_{0}^{t} \varphi_{q}\left(\int_{s}^{1} f(\tau, u(\tau)) \mathrm{d} \tau\right) \mathrm{d} s+\sum_{k=1}^{m} H\left(t, t_{k}\right) I_{k}\left(u\left(t_{k}\right)\right)\right]^{p^{*}} \\
& \geq \kappa_{1}\left[\int_{0}^{t} \varphi_{q}\left(\int_{s}^{1} f(\tau, u(\tau)) \mathrm{d} \tau\right) \mathrm{d} s\right]^{p^{*}}+\kappa_{1}\left[\sum_{k=1}^{m} H\left(t, t_{k}\right) I_{k}\left(u\left(t_{k}\right)\right)\right]^{p^{*}}
\end{aligned}
$$




$$
\begin{aligned}
& \geq \kappa_{1} \int_{0}^{t} \int_{s}^{1} f^{\frac{p_{s}}{p-1}}(\tau, u(\tau)) \mathrm{d} \tau \mathrm{d} s+\kappa_{2} \sum_{k=1}^{m} H^{p^{*}}\left(t, t_{k}\right) I_{k}^{p^{*}}\left(u\left(t_{k}\right)\right) \\
& \geq \kappa_{1} \int_{0}^{1} G(t, s) f^{\frac{p^{*}}{p^{-1}}}(s, u(s)) \mathrm{d} s+\kappa_{2} \sum_{k=1}^{m} H\left(t, t_{k}\right) I_{k}^{p^{*}}\left(u\left(t_{k}\right)\right) .
\end{aligned}
$$

Multiply both sides of (3.5) by $\sin \left(\frac{\pi t}{2}\right)$ and then integrate over $[0,1]$ and use (2.5) to obtain

$$
\begin{aligned}
\int_{0}^{1} u^{p^{*}}(t) \sin \left(\frac{\pi t}{2}\right) \mathrm{d} t \geq & \kappa_{1} \int_{0}^{1} \sin \left(\frac{\pi t}{2}\right) \int_{0}^{1} G(t, s) f^{\frac{p^{*}}{p^{-1}}}(s, u(s)) \mathrm{d} s \mathrm{~d} t \\
& +\kappa_{2} \sum_{k=1}^{m} \int_{0}^{1} \sin \left(\frac{\pi t}{2}\right) H\left(t, t_{k}\right) I_{k}^{p^{*}}\left(u\left(t_{k}\right)\right) \mathrm{d} t \\
\geq & \frac{4 \kappa_{1}}{\pi^{2}} \int_{0}^{1} f^{\frac{p^{*}}{p^{*}}}(t, u(t)) \sin \left(\frac{\pi t}{2}\right) \mathrm{d} t \\
& +\frac{2 \kappa_{2}}{\pi} \sum_{k=1}^{m} I_{k}^{p^{*}}\left(u\left(t_{k}\right)\right) \cos \left(\frac{\pi t_{k}}{2}\right) .
\end{aligned}
$$

The above and (H1) imply that

$$
\begin{aligned}
\int_{0}^{1} u^{p_{*}^{*}}(t) \sin \left(\frac{\pi t}{2}\right) \mathrm{d} t \geq & \frac{4 a_{1}^{p_{*}^{p-1}} \kappa_{1}}{\pi^{2}} \int_{0}^{1} u^{p_{*}}(t) \sin \left(\frac{\pi t}{2}\right) \mathrm{d} t \\
& +\frac{2 a_{2}^{p_{*}^{*}} \kappa_{2}}{\pi} \sum_{k=1}^{m} u^{p_{*}^{*}}\left(t_{k}\right) \cos \left(\frac{\pi t_{k}}{2}\right) .
\end{aligned}
$$

By (3.7), we have $\frac{4 a_{1}^{\frac{p *}{p-1}} \kappa 1}{\pi^{2}} \leq 1$. If $\frac{4 a_{1}^{\frac{p_{s}}{p-1}} \kappa 1}{\pi^{2}}=1$, then $u\left(t_{k}\right) \equiv 0, k=1,2, \ldots, m$, and in view of the concavity and the nondecreasing nature of $u$, we find $u(t) \equiv 0,0 \leq t \leq 1$, contradicting $u \in P \cap \partial B_{r}$. So, $\frac{4 a_{1}^{\frac{p s}{p-1}} \kappa_{1}}{\pi^{2}}<1$.

Since $u \in P \cap \partial B_{r}, u^{p^{*}}(t) \leq\|u\|^{p^{*}}=r^{p^{*}}$. Therefore,

$$
\int_{0}^{1} u^{p^{*}}(t) \sin \left(\frac{\pi t}{2}\right) \mathrm{d} t \leq r^{p^{*}} \int_{0}^{1} \sin \left(\frac{\pi t}{2}\right) \mathrm{d} t=\frac{2 r^{p^{*}}}{\pi} .
$$

Combining (3.7) and (2.2), we obtain

$$
\frac{2\left(\pi^{2}-4 a_{1}^{\frac{p^{*}}{p-1}} \kappa_{1}\right) r^{p^{*}}}{\pi^{3}} \geq \frac{2 a_{2}^{p^{*}} \kappa_{2} r^{p^{*}} t_{1}^{p_{*}}}{\pi} \sum_{k=1}^{m} \cos \left(\frac{\pi t_{k}}{2}\right) .
$$

Therefore, $4 a_{1}^{\frac{p^{*}}{p-1}} \kappa_{1}+\pi^{2} a_{2}^{p^{*}} t_{1}^{p^{*}} \kappa_{2} \sum_{k=1}^{m} \cos \left(\frac{\pi t_{k}}{2}\right) \leq \pi^{2}$, which contradicts (H1). Thus we have

$$
u \nsupseteq A u, \quad \text { for any } u \in P \cap \partial B_{r} .
$$

On the other hand, by $(\mathrm{H} 2)$, we shall prove that there exists a sufficiently large number $R>0$ such that $u \not \subset A u, \forall u \in P \cap \partial B_{R}$. Suppose there exists $u \in P \cap \partial B_{R}$ such that $u \leq A u$. 
This, together with Lemma 2.4, yields

$$
\begin{aligned}
u^{p^{*}}(t) & \leq\left[\int_{0}^{t} \varphi_{q}\left(\int_{s}^{1} f(\tau, u(\tau)) \mathrm{d} \tau\right) \mathrm{d} s+\sum_{k=1}^{m} H\left(t, t_{k}\right) I_{k}\left(u\left(t_{k}\right)\right)\right]^{p^{*}} \\
& \leq \kappa_{3}\left[\int_{0}^{t} \varphi_{q}\left(\int_{s}^{1} f(\tau, u(\tau)) \mathrm{d} \tau\right) \mathrm{d} s\right]^{p^{*}}+\kappa_{3}\left[\sum_{k=1}^{m} H\left(t, t_{k}\right) I_{k}\left(u\left(t_{k}\right)\right)\right]^{p^{*}} \\
& \leq \kappa_{3} \int_{0}^{t} \int_{s}^{1} f^{\frac{p^{*}}{p-1}}(\tau, u(\tau)) \mathrm{d} \tau \mathrm{d} s+\kappa_{4} \sum_{k=1}^{m} H^{p^{*}}\left(t, t_{k}\right) I_{k}^{p^{*}}\left(u\left(t_{k}\right)\right) \\
& \leq \kappa_{3} \int_{0}^{1} G(t, s) f^{\frac{p^{*}}{p-1}}(s, u(s)) \mathrm{d} s+\kappa_{4} \sum_{k=1}^{m} H\left(t, t_{k}\right) I_{k}^{p^{*}}\left(u\left(t_{k}\right)\right) .
\end{aligned}
$$

Multiply both sides of the above by $\sin \left(\frac{\pi t}{2}\right)$ and integrate over $[0,1]$ and use (2.5) to obtain

$$
\begin{aligned}
\int_{0}^{1} u^{p^{*}}(t) \sin \left(\frac{\pi t}{2}\right) \mathrm{d} t \leq & \kappa_{3} \int_{0}^{1} \sin \left(\frac{\pi t}{2}\right) \int_{0}^{1} G(t, s) f^{\frac{p^{*}}{p-1}}(s, u(s)) \mathrm{d} s \mathrm{~d} t \\
& +\kappa_{4} \sum_{k=1}^{m} \int_{0}^{1} \sin \left(\frac{\pi t}{2}\right) H\left(t, t_{k}\right) I_{k}^{p^{*}}\left(u\left(t_{k}\right)\right) \mathrm{d} t \\
\leq & \frac{4 \kappa_{3}}{\pi^{2}} \int_{0}^{1} f^{\frac{p^{*}}{p-1}}(t, u(t)) \sin \left(\frac{\pi t}{2}\right) \mathrm{d} t \\
& +\frac{2 \kappa_{4}}{\pi} \sum_{k=1}^{m} I_{k}^{p^{*}}\left(u\left(t_{k}\right)\right) \cos \left(\frac{\pi t_{k}}{2}\right) .
\end{aligned}
$$

Combining this and (H2), we get

$$
\begin{aligned}
\int_{0}^{1} u^{p^{*}}(t) \sin \left(\frac{\pi t}{2}\right) \mathrm{d} t \leq & \frac{4 \kappa_{3}}{\pi^{2}} \int_{0}^{1}\left(b_{1} u^{p-1}(t)+c\right)^{\frac{p^{*}}{p-1}} \sin \left(\frac{\pi t}{2}\right) \mathrm{d} t \\
& +\frac{2 \kappa_{4}}{\pi} \sum_{k=1}^{m}\left(b_{2} u\left(t_{k}\right)+c\right)^{p^{*}} \cos \left(\frac{\pi t_{k}}{2}\right) \\
\leq & \frac{4 b_{1}^{\frac{p^{*}}{p-1}} \kappa_{5}}{\pi^{2}} \int_{0}^{1} u^{p^{*}}(t) \sin \left(\frac{\pi t}{2}\right) \mathrm{d} t \\
& +\frac{2 b_{2}^{p^{*}} \kappa_{6}}{\pi} \sum_{k=1}^{m} u^{p^{*}}\left(t_{k}\right) \cos \left(\frac{\pi t_{k}}{2}\right)+c_{0},
\end{aligned}
$$

where $c_{0}=\frac{8 c^{\frac{p^{n}}{p-1}} \kappa_{5}}{\pi^{3}}+\frac{2 c^{p^{*}} \kappa_{6}}{\pi} \sum_{k=1}^{m} \cos \left(\frac{\pi t_{k}}{2}\right)$. Consequently, by (2.2) we have

$$
\begin{aligned}
\|u\|^{p^{*}}\left(\pi^{2}-4 b_{1}^{\frac{p^{*}}{p-1}} \kappa_{5}\right) \int_{0}^{1} t^{p^{*}} \sin \left(\frac{\pi t}{2}\right) \mathrm{d} t & \leq\left(\pi^{2}-4 b_{1}^{\frac{p^{*}}{p-1}} \kappa_{5}\right) \int_{0}^{1} u^{p^{*}} \sin \left(\frac{\pi t}{2}\right) \mathrm{d} t \\
& \leq 2 \pi b_{2}^{p^{*}} \kappa_{6}\|u\|^{p^{*}} \sum_{k=1}^{m} \cos \left(\frac{\pi t_{k}}{2}\right)+\pi^{2} c_{0} .
\end{aligned}
$$


Therefore,

$$
\|u\|^{p^{*}} \leq \frac{\pi^{2} c_{0}}{\left(\pi^{2}-4 b_{1}^{\frac{p^{*}}{p-1}} \kappa_{5}\right) \int_{0}^{1} t^{p^{*}} \sin \left(\frac{\pi t}{2}\right) \mathrm{d} t-2 \pi b_{2}^{p^{*}} \kappa_{6} \sum_{k=1}^{m} \cos \left(\frac{\pi t_{k}}{2}\right)}:=K_{1}>0 .
$$

Choosing $R>\sqrt[p^{*}]{K_{1}}$ and $R>r$, we have

$u \not \subset A u, \quad$ for all $u \in P \cap \partial B_{R}$.

Therefore, (3.8) and (3.12), together with Lemma 2.3, guarantee that (1.3) has at least one positive solution in $\left(B_{R} \backslash \bar{B}_{r}\right) \cap P$.

Theorem 3.2 Suppose that (H3)-(H4) are satisfied. Then (1.3) has at least one positive solution.

Proof If (H3) is satisfied, we will prove that there exists a sufficiently large number $R>0$ such that $u \nsupseteq A u, \forall u \in P \cap \partial B_{R}$. Suppose there exists $u \in P \cap \partial B_{R}$ such that $u \geq A u$, and then

$$
u(t) \geq \int_{0}^{t} \varphi_{q}\left(\int_{s}^{1} f(\tau, u(\tau)) \mathrm{d} \tau\right) \mathrm{d} s+\sum_{k=1}^{m} H\left(t, t_{k}\right) I_{k}\left(u\left(t_{k}\right)\right) .
$$

In view of $0<\frac{p_{*}}{p-1} \leq 1$, from (3.3), we know $\left(a_{3} u^{p-1}\right)^{\frac{p_{*}}{p-1}} \leq(f+c)^{\frac{p_{*}}{p-1}} \leq f^{\frac{p^{*}}{p-1}}+c^{\frac{p^{*}}{p-1}}$. Accordingly, $f^{\frac{p^{*}}{p^{2}}} \geq\left(a_{3} u^{p-1}\right)^{\frac{p^{*}}{p-1}}-c^{\frac{p^{*}}{p-1}}$. Similarly, $I_{k}^{p_{*}} \geq\left(a_{4} u\right)^{p^{*}}-c^{p^{*}}$. These and (3.6) imply that

$$
\begin{aligned}
\int_{0}^{1} u^{p_{*}}(t) \sin \left(\frac{\pi t}{2}\right) \mathrm{d} t \geq & \frac{4 \kappa_{1}}{\pi^{2}} \int_{0}^{1}\left[\left(a_{3} u^{p-1}(t)\right)^{\frac{p^{*}}{p-1}}-c^{\frac{p^{s}}{p-1}}\right] \sin \left(\frac{\pi t}{2}\right) \mathrm{d} t \\
& +\frac{2 \kappa_{2}}{\pi} \sum_{k=1}^{m}\left[\left(a_{4} u\left(t_{k}\right)\right)^{p^{*}}-c^{p^{*}}\right] \cos \left(\frac{\pi t_{k}}{2}\right) \\
\geq & \frac{4 a_{3}^{p^{p}-1} \kappa_{1}}{\pi^{2}} \int_{0}^{1} u^{p^{*}}(t) \sin \left(\frac{\pi t}{2}\right) \mathrm{d} t \\
& +\frac{2 a_{4}^{p^{*}} \kappa_{2}}{\pi} \sum_{k=1}^{m} u^{p^{*}}\left(t_{k}\right) \cos \left(\frac{\pi t_{k}}{2}\right)-c_{1},
\end{aligned}
$$

where $c_{1}=\frac{8 c^{\frac{p_{s}}{p-1}} \kappa_{1}}{\pi^{3}}+\frac{2 \kappa_{2} c^{p_{s}}}{\pi} \sum_{k=1}^{m} \cos \left(\frac{\pi t_{k}}{2}\right)$.

Now, we consider two cases.

Case 1. If $1 \geq \frac{4 a_{3}^{\frac{p^{*}}{p-1}} \kappa}{\pi^{2}}$, by (2.2) we obtain

$$
\begin{aligned}
\frac{2\left(\pi^{2}-4 a_{3}^{\frac{p_{s}}{p^{2}}} \kappa_{1}\right)\|u\|^{p_{*}}}{\pi} & \geq\left(\pi^{2}-4 a_{3}^{\frac{p^{p}}{p-1}} \kappa_{1}\right) \int_{0}^{1} u^{p_{*}}(t) \sin \left(\frac{\pi t}{2}\right) \mathrm{d} t \\
& \geq 2 \pi a_{4}^{p_{*}^{*}} t_{1}^{p_{*}} \kappa_{2}\|u\|^{p^{*}} \sum_{k=1}^{m} \cos \left(\frac{\pi t_{k}}{2}\right)-\pi^{2} c_{1},
\end{aligned}
$$


i.e.,

$$
\|u\|^{p^{*}} \leq \frac{\frac{\pi^{3} c_{1}}{2}}{4 a_{3}^{\frac{p^{*}}{p-1}} \kappa_{1}+\pi^{2} a_{4}^{p^{*}} t_{1}^{p^{*}} \kappa_{2} \sum_{k=1}^{m} \cos \left(\frac{\pi t_{k}}{2}\right)-\pi^{2}}:=K_{2}>0 .
$$

Case 2. If $1<\frac{4 a_{3}^{\frac{p}{p-1}} \kappa_{1}}{\pi^{2}}$, by $(2.2)$ we have

$$
c_{1} \pi^{2} \geq\left(4 a_{3}^{\frac{p^{*}}{p-1}} \kappa_{1}-\pi^{2}\right)\|u\|^{p^{*}} \int_{0}^{1} t^{p^{\prime \prime}} \sin \left(\frac{\pi t}{2}\right) \mathrm{d} t+2 \pi t_{1}^{p^{*}}\|u\|^{p^{*}} a_{4}^{p^{\prime \prime}} \kappa_{2} \sum_{k=1}^{m} \cos \left(\frac{\pi t_{k}}{2}\right) .
$$

In view of $\frac{4}{\pi^{2}} \leq \int_{0}^{1} t^{p^{*}} \sin \left(\frac{\pi t}{2}\right) \mathrm{d} t \leq \frac{2}{\pi}$, we obtain

$$
\|u\|^{p^{*}} \leq \frac{\frac{\pi^{4} c_{1}}{4}}{4 a_{3}^{\frac{p^{*}}{p-1}} \kappa_{1}+\frac{\pi^{3}}{2} a_{4}^{p_{*}} t_{1}^{p_{s}} \kappa_{2} \sum_{k=1}^{m} \cos \left(\frac{\pi t_{k}}{2}\right)-\pi^{2}}:=K_{3}>0 .
$$

Choosing $R>\max \left\{\sqrt[p *]{K_{2}}, \sqrt[p p]{K_{3}}, r\right\}$ ( $r$ is determined by $(\mathrm{H} 4)$ ), we get

$$
u \nsupseteq A u, \quad \forall u \in P \cap \partial B_{R} .
$$

On the other hand, if (H4) is satisfied, then $u \not \leq A u, \forall u \in P \cap \partial B_{r}$. If not, there exists $u \in P \cap \partial B_{r}$ such that $u \leq A u$. It follows from (3.10) and (H4) that

$$
\begin{aligned}
\int_{0}^{1} u^{p^{*}}(t) \sin \left(\frac{\pi t}{2}\right) \mathrm{d} t \leq & \frac{4 \kappa_{3}}{\pi^{2}} \int_{0}^{1}\left(b_{3} u^{p-1}(t)\right)^{\frac{p^{*}}{p-1}} \sin \left(\frac{\pi t}{2}\right) \mathrm{d} t \\
& +\frac{2 \kappa_{4}}{\pi} \sum_{k=1}^{m}\left(b_{4} u\left(t_{k}\right)\right)^{p^{*}} \cos \left(\frac{\pi t_{k}}{2}\right) \\
\leq & \frac{4 b_{3}^{\frac{p^{n}}{p-1}} \kappa_{3}}{\pi^{2}} \int_{0}^{1} u^{p^{*}}(t) \sin \left(\frac{\pi t}{2}\right) \mathrm{d} t \\
& +\frac{2 b_{4}^{p^{*}} \kappa_{4}}{\pi} \sum_{k=1}^{m} u^{p^{*}}\left(t_{k}\right) \cos \left(\frac{\pi t_{k}}{2}\right) .
\end{aligned}
$$

Therefore,

$$
\begin{aligned}
\|u\|^{p^{*}}\left(\pi^{2}-4 b_{3}^{\frac{p^{*}}{p-1}} \kappa_{3}\right) \int_{0}^{1} t^{p^{*}} \sin \left(\frac{\pi t}{2}\right) \mathrm{d} t & \leq\left(\pi^{2}-4 b_{3}^{\frac{p^{*}}{p-1}} \kappa_{3}\right) \int_{0}^{1} u^{p^{*}} \sin \left(\frac{\pi t}{2}\right) \mathrm{d} t \\
& \leq 2 \pi b_{4}^{p^{*}} \kappa_{4}\|u\|^{p^{*}} \sum_{k=1}^{m} \cos \left(\frac{\pi t_{k}}{2}\right),
\end{aligned}
$$

i.e.,

$$
4 b_{3}^{\frac{p^{*}}{p-1}} \kappa_{3}+\frac{2 \pi b_{4}^{p^{*}} \kappa_{4} \sum_{k=1}^{m} \cos \left(\frac{\pi t_{k}}{2}\right)}{\int_{0}^{1} t^{p^{*}} \sin \left(\frac{\pi t}{2}\right) \mathrm{d} t} \geq \pi^{2}
$$


which contradicts (H4). Thus

$$
u \nless A u, \quad \forall u \in \partial B_{r} \cap P .
$$

By Lemma 2.3, (3.16) and (3.18) imply that (1.3) has at least one positive solution in $\left(B_{R} \backslash\right.$ $\left.\bar{B}_{r}\right) \cap P$.

Theorem 3.3 Suppose that (H1), (H3) and (H5) are satisfied. Then (1.3) has at least two positive solutions.

Proof If $u \in P \cap \partial B_{\rho}$, it follows from (H5) that

$$
\begin{aligned}
\|A u\| & \leq \int_{0}^{1} \varphi_{q}\left(\int_{s}^{1} f(\tau, u(\tau)) \mathrm{d} \tau\right) \mathrm{d} s+\sum_{k=1}^{m} I_{k}\left(u\left(t_{k}\right)\right) \\
& \leq \int_{0}^{1} \varphi_{q}\left(\int_{s}^{1} \eta^{p-1} \rho^{p-1} \mathrm{~d} \tau\right) \mathrm{d} s+\sum_{k=1}^{m} \rho \eta_{k} \\
& \leq \rho\left(\eta \frac{p-1}{p}+\sum_{k=1}^{m} \eta_{k}\right)<\rho\left(\eta+\sum_{k=1}^{m} \eta_{k}\right) \leq\|u\|,
\end{aligned}
$$

from which we obtain

$$
u \not \subset A u, \quad \forall u \in \partial B \rho \cap P .
$$

On the other hand, by (H1) and (H3), we may take $0<r<\rho$ and $R>\rho$ such that $u \nsupseteq A u$, $\forall u \in P \cap \partial B_{r}$ and $u \nsupseteq A u, \forall u \in P \cap \partial B_{R}$ (see the proofs of Theorems 3.1 and 3.2). Now Lemma 2.3 guarantees that the operator $A$ has at least two fixed points, one in $\left(B_{R} \backslash \bar{B}_{\rho}\right) \cap P$ and the other in $\left(B_{\rho} \backslash \bar{B}_{r}\right) \cap P$. The proof is completed.

Theorem 3.4 Suppose that (H2), (H4) and (H6) are satisfied. Then (1.3) has at least two positive solutions.

Proof For any $u \in P \cap \partial B_{\rho}, u(t) \geq t_{1}\|u\|=t_{1} \rho$ for all $t \in\left[t_{1}, 1\right]$. It follows from (H6) that

$$
\begin{aligned}
\|A u\| & =\int_{0}^{1} \varphi_{q}\left(\int_{s}^{1} f(\tau, u(\tau)) \mathrm{d} \tau\right) \mathrm{d} s+\sum_{k=1}^{m} I_{k}\left(u\left(t_{k}\right)\right) \\
& \geq \int_{t_{1}}^{1} \varphi_{q}\left(\int_{s}^{1} f(\tau, u(\tau)) \mathrm{d} \tau\right) \mathrm{d} s+\sum_{k=1}^{m} I_{k}\left(u\left(t_{k}\right)\right) \\
& \geq \int_{t_{1}}^{1} \varphi_{q}\left(\int_{s}^{1} \eta^{p-1} \rho^{p-1} \mathrm{~d} \tau\right) \mathrm{d} s+\sum_{k=1}^{m} \eta_{k} \rho \\
& \geq \rho\left(\eta p^{-1}(p-1)\left(1-t_{1}\right)^{p /(p-1)}+\sum_{k=1}^{m} \eta_{k}\right)>\rho=\|u\| .
\end{aligned}
$$

Thus, 
On the other hand, by (H2) and (H4), we may take $0<r<\rho$ and $R>\rho$ such that $u \not \subset A u$, $\forall u \in P \cap \partial B_{r}$ and $u \not \leq A u, \forall u \in P \cap \partial B_{R}$ (see the proofs of Theorems 3.1 and 3.2). Thus Lemma 2.3 indicates that the operator $A$ has at least two fixed points, one in $\left(B_{R} \backslash \bar{B}_{\rho}\right) \cap P$ and the other in $\left(B_{\rho} \backslash \bar{B}_{r}\right) \cap P$. The proof is completed.

\section{Examples}

Example 4.1 Consider the impulsive boundary value problem

$$
\left\{\begin{array}{l}
\left(\varphi_{p}\left(u^{\prime}\right)\right)^{\prime}=-f(t, u), \quad t \neq \frac{1}{2}, 0<t<1, \\
\left.\Delta u\right|_{t=\frac{1}{2}}=I_{1}\left(u\left(\frac{1}{2}\right)\right), \\
\left.\Delta u^{\prime}\right|_{t=\frac{1}{2}}=0 \\
u(0)=u^{\prime}(1)=0
\end{array}\right.
$$

where $p=\frac{3}{2}$.

Case 1. Let $f(t, u)=u^{\alpha}, 0<\alpha<\frac{1}{2}, I_{1}(u)=u^{\alpha_{1}}, 0<\alpha_{1}<1$. Then

$$
\lim _{u \rightarrow 0^{+}} \frac{f(t, u)}{u^{p-1}}=\lim _{u \rightarrow 0^{+}} \frac{u^{\alpha}}{u^{p-1}}=\infty, \quad \lim _{u \rightarrow 0^{+}} \frac{I_{1}(u)}{u}=\lim _{u \rightarrow 0^{+}} u^{\alpha_{1}-1}=\infty .
$$

From (4.2) we see that (H1) is satisfied. In fact, since $p=3 / 2$ and $t_{1}=1 / 2, p^{*}=1 / 2, \kappa_{1}=\kappa_{2}=$ $1 / \sqrt{2}$. Taking $a_{1}=3, a_{2}=1$, we get

$$
a_{1}^{\frac{p^{\infty}}{p-1}} \kappa_{1}+\frac{\pi^{2}}{4} a_{2}^{p^{n}} t_{1}^{p^{*}} \kappa_{2} \sum_{k=1}^{m} \cos \left(\frac{\pi t_{k}}{2}\right)>\frac{\pi^{2}}{4} .
$$

Moreover,

$$
\lim _{u \rightarrow \infty} \frac{f(t, u)}{u^{p-1}}=\lim _{u \rightarrow \infty} \frac{u^{\alpha}}{u^{p-1}}=0, \quad \lim _{u \rightarrow \infty} \frac{I_{1}(u)}{u}=\lim _{u \rightarrow \infty} u^{\alpha_{1}-1}=0 .
$$

From (4.3) we see that ( $\mathrm{H} 2)$ is satisfied, for example taking $b_{1}=1, b_{2}=\frac{1}{10}$. All the assumptions in Theorem 3.1 are satisfied, and the problem (4.1) has at least one positive solution by Theorem 3.1 .

Case 2 . Take $f(t, u)=u^{\beta}, \beta>\frac{1}{2}, I_{1}(u)=u^{\beta_{1}}, \beta_{1}>1$. One can easily verify conditions (H3) and (H4). Thus the problem (4.1) has at least one positive solution by Theorem 3.2.

Case 3. Let $f(t, u)=\frac{u^{\alpha}+u^{\beta}}{5}, 0<\alpha<\frac{1}{2}<\beta, I_{1}(u)=\frac{u}{5}$. Thus, we get

$$
\lim _{u \rightarrow 0^{+}} \frac{f(t, u)}{u^{p-1}}=\lim _{u \rightarrow 0^{+}} \frac{u^{\alpha}+u^{\beta}}{5 u^{p-1}}=\infty .
$$

From (4.4) we see that (H1) is satisfied. Note

$$
\lim _{u \rightarrow \infty} \frac{f(t, u)}{u^{p-1}}=\lim _{u \rightarrow \infty} \frac{u^{\alpha}+u^{\beta}}{5 u^{p-1}}=\infty .
$$

From (4.5) we see that (H3) is satisfied. Take $\rho=1, \eta=\frac{4}{5}, \eta_{1}=\frac{1}{5}$ in (H5), and note for $0 \leq u \leq \rho$ and $t \in[0,1]$ that $f(t, u) \leq \frac{\rho^{\alpha}+\rho^{\beta}}{5}=\frac{2}{5}<\frac{2 \sqrt{5}}{5}=\eta^{\frac{1}{2}}, I_{1}(u)=\frac{u}{5} \leq \frac{1}{5}=\eta_{1}$. As a result, (H5) holds. From Theorem 3.3, the problem (4.1) has at least two positive solutions. 


\section{Competing interests}

The authors declare that they have no competing interests.

\section{Authors' contributions}

The first author conceived of the study and carried out the proof. Authors read and approved the final manuscript.

\section{Author details}

'Department of Mathematics, Shandong Jianzhu University, Jinan, Shandong 250101, China. ${ }^{2}$ Department of Mathematics, National University of Ireland, Galway, Ireland. ${ }^{3}$ School of Mathematics, Shandong University, Jinan, Shandong 250100, China.

\section{Acknowledgements}

The authors are grateful to the anonymous referee for his/her valuable suggestions. The first author thanks Prof. Zhongli Wei for his valuable help. This work is supported financially by the Shandong Provincial Natural Science Foundation (ZR2012AQ007) and Graduate Independent Innovation Foundation of Shandong University (yzc12063).

Received: 18 April 2012 Accepted: 28 August 2012 Published: 11 September 2012

\section{References}

1. Glowinski, R, Rappaz, J: Approximation of a nonlinear elliptic problem arising in a non-Newtonian fluid flow model in glaciology. Math. Model. Numer. Anal. 37, 175-186 (2003)

2. Diaz, J, de Thélin, F: On a nonlinear parabolic problem arising in some models related to turbulent flows. SIAM J. Math. Anal. 25, 1085-1111 (1994)

3. Lian, H, Ge, WG: Positive solutions for a four-point boundary value problem with the $p$-Laplacian. Nonlinear Anal. 68, 3493-3503 (2008)

4. Zhang, X, Ge, W: Impulsive boundary value problems involving the one-dimensional p-Laplacian. Nonlinear Anal. 70 1692-1701 (2009)

5. Cabada, A, Tomeček, J: Extremal solutions for nonlinear functional $p$-Laplacian impulsive equations. Nonlinear Anal. 67, 827-841 (2007)

6. Xu, J, Kang, P, Wei, Z: Singular multipoint impulsive boundary value problem with $p$-Laplacian operator. J. Appl. Math. Comput. 30, 105-120 (2009)

7. Feng, M: Multiple positive solutions of four-order impulsive differential equations with integral boundary conditions and one-dimensional p-Laplacian. Bound. Value Probl. (2011). doi:10.1155/2011/654871

8. Li, $\mathrm{P}, \mathrm{Chen}, \mathrm{H}, \mathrm{Wu}, \mathrm{Y}$ : Multiple positive solutions of $n$-point boundary value problems for $p$-Laplacian impulsive dynamic equations on time scales. Comput. Math. Appl. 60, 2572-2582 (2010)

9. Zhang, X: Existence and successive iteration of positive solutions for some impulsive multi-point boundary value problem with $p$-Laplacian on infinite interval. J. Syst. Sci. Math. Sci. 30, 619-632 (2010)

10. Yang, Z: Positive solutions for a system of $p$-Laplacian boundary value problems. Comput. Math. Appl. 62, 4429-4438 (2011)

11. Bai, L, Dai, B: Three solutions for a $p$-Laplacian boundary value problem with impulsive effects. Appl. Math. Comput 217, 9895-9904 (2011)

12. Yang, Z, O'Regan, D: Positive solutions for one-dimensional $p$-Laplacian equations. Math. Comput. Model. 55 1942-1950 (2012)

13. Xu, J, Yang, Z: Positive solutions for a fourth order $p$-Laplacian boundary value problem. Nonlinear Anal. 74, 2612-2623 (2011)

14. Guo, D, Lakshmikantham, V: Nonlinear Problems in Abstract Cones. Academic Press, Orlando (1988)

doi:10.1186/1687-1847-2012-159

Cite this article as: Ding and $O^{\prime}$ Regan: Positive solutions for a second-order $p$-Laplacian impulsive boundary value problem. Advances in Difference Equations 2012 2012:159.

\section{Submit your manuscript to a SpringerOpen ${ }^{\circ}$ journal and benefit from:}

- Convenient online submission

Rigorous peer review

- Immediate publication on acceptance

- Open access: articles freely available online

- High visibility within the field

- Retaining the copyright to your article 